\title{
Optimal Resources for Children's Surgical Care: Executive Summary
}

\author{
Global Initiative for Children's Surgery
}

Global Initiative for Children's Surgery Collaborating Authors:

David Grabski, Emmanuel Ameh, Doruk Ozgediz, Keith Oldham, Francis A. Abantanga, Mohamed Abdelmalak, Nurudeen Abdulraheem, Niyi Ade-Ajayi, Edna Adan Ismail, Adesoji Ademuyiwa, Eltayeb Ahmed, Sunday Ajike, Olugbemi Benedict Akintububo, Felix Alakaloko, Brendan Allen, Vanda Amado, Shanthi Anbuselvan, Jamie Anderson, Theophilus Teddy Kojo Anyomih, Leopold Asakpa, Gudeta Assegie, Jason Axt, Ruben Ayala, Frehun Ayele, Harshjeet Singh Bal, Rouma Bankole, Tahmina Banu, Tim Beacon, Zaitun Bokhari, Hiranya Kumar Borah, Eric Borgstein, Nick Boyd, Jason Brill, Britta Budde-Schwartzman, Fred Bulamba, Marilyn Butler, Bruce Bvulani, Sarah Cairo, Juan Francisco Campos Rodezno, Massimo Caputo, Milind Chitnis, Maija Cheung, Bruno Cigliano, Damian Clarke, Michael Cooper, Tessa Concepcion, Scott Corlew, David Cunningham, Sergio D’Agostino, Shukri Dahir, Bailey Deal, Miliard Derbew, Sushil Dhungel, David Drake, Elizabeth Drum, Bassey Edem, Stella Eguma, Olumide Elebute, Beda R. Espineda, Samuel Espinoza, Faye Evans, Omolara Faboya, Jacques Fadhili Bake, Tatiana Fazecas, Mohammad Rafi Fazli, Graham Fieggen, Anthony Figaji, Jean Louis Fils, Tamara Fitzgerald, Randall Flick, Gacelle Fossi, George Galiwango, Lily Saldaña Gallo, Mike Ganey, Zipporah Gathuya, Maryam Ghavami Adel, Vafa Ghorban Sabagh, Sridhar Gibikote, Hetal Gohil, Laura Goodman, Sarah Greenberg, Russell Gruen, Lars Hagander, Rahimullah Hamid, Erik Hansen, William Harkness, Mauricio Herrera, Intisar Hisham, Andrew Hodges, Sarah Hodges, Ai Xuan Holterman, Andrew Howard, Romeo Ignacio, Dawn Ireland, Enas Ismail, Rebecca Jacob, Anette Jacobsen, Zahra Jaffry, Deeptiman James, Ebor Jacob James, Adiyasuren Jamiyanjav, Kathy Jenkins, Guy Jensen, Maria Jimenez, Tarun John K Jacob, Walter Johnson, Anita Joselyn, Nasser Kakembo, Patrick Kamalo, Neema Kaseje, Bertille Ki, Phyllis Kisa, Peter Kim, Krishna Kumar, Rashmi Kumar, Charlotte Kvasnovsky, Ananda Lamahewage, Monica Langer, Christopher Lavy, Taiwo Lawal, Colin Lazarus, Andrew Leather, Chelsea Lee, Basil Leodoro, Allison Linden, Katrine Lofberg, Jonathan Lord, Jerome Loveland, Leecarlo Millano Lumban Gaol, Vrisha Madhuri, Pavrette Magdala, Luc Kalisya Malemo, Aeesha Malik, John Mathai, Marcia Matias, Bothwell Mbuwayesango, Merrill McHoney, Liz McLeod, Ashish Minocha, Charles Mock, Mubarak Mohamed, Ivan Molina, Ashika Morar, Zahid Mukhtar, Mulewa Mulenga, Bhargava Mullapudi, Jack Mulu, Byambajav Munkhjargal, Arlene Muzira, Mary Nabukenya, Mark Newton, Jessica Ng, Karissa Nguyen, Laurence Isaaya Ntawunga, Peter M. Nthumba, Alp Numanoglu, Benedict Nwomeh, Kristin Ojomo, Maryrose Osazuwa, Emmanuel Owusu Abem, Shazia Peer, Norgrove Penny, Dan Poenaru, Robin Petroze, Vithya Priya, Ekta Rai, Lola Raji, Vinitha Paul Ravindran, Desigen Reddy, Henry Rice, Yona Ringo, Amezene Robelie, Jose Roberto Baratella, David Rothstein, Coleen Sabatini, Soumitra Saha, Saurabh Saluja, Lubna Samad, John Sekabira, Justina Seyi-Olajide, Bello B. Shehu, Sabina Siddiqui, Ritesh Shrestha, David Sigalet, Martin Situma, Emily Smith, Adrienne Socci, David Spiegel, Peter Ssenyonga, Jacob Stephenson, Richard Stewart, Erin Stieber, Etienne St-Louis, Vinayak Shukla, Thomas Sims, Faustin Felicien Mouafo Tambo, Robert Tamburro, Mansi Tara, Ahmad Tariq, Reju Thomas, Leopold Torres Contreras, Stephen Ttendo, Benno Ure, Luca Vricella, Luis Vasquez, Vijayakumar Raju, Jorge Villacis, Gustavo Villanova, Catherine deVries, Amira Waheeb, Saber Waheeb, Albert Wandaogo, Anne Wesonga, Omolara Williams, Sigal Willner, Nyo Nyo Win, Hussein Wissanji, Paul Mwindekuma Wondoh, Garreth Wood, Naomi Wright, Benjamin Yapo, George Youngson, Yasmine Yousef, Denléwendé Sylvain Zabsonre, Luis Enrique Zea Salazar, Adiyasuren Zevee, Bistra Zheleva, Kokila Lakhoo, Diana Farmer, Stephen Bickler 
Corresponding author:

Stephen W. Bickler, MD, FACS, FAAP

Division of Pediatric Surgery

Rady Children's Hospital—University of California San Diego

3030 Children's Way, MC 5136

San Diego, CA 92123 USA 


\section{Executive Summary}

There is growing recognition that safe surgery and anesthesia should be an essential component of universal health care in low- and middle-income countries (LMICs). Landmark studies in 2015, by the Disease Control Priorities Project (DCP3) and Lancet Commission on Global Surgery (LCoGS) were important in defining the scale and scope of the surgical burden worldwide, priority surgical interventions, and economic impact of surgical care ${ }^{1,2}$. Shortly after the release of their two reports, the World Health Assembly passed resolution 68/15 designed to strengthen emergency and essential surgical and anesthesia care as a component of universal health coverage ${ }^{3}$. To facilitate implementation of the DCP3 and LCoGS recommendations a growing number of countries are developing national surgical, obstetric and anesthesia plans ${ }^{4}$. National surgical plans are an iterative process where stakeholders use country-level data to develop a contextually relevant and sustainable plan to ensure that surgical and obstetrical services are available for an entire country or region.

Of the 4.8 billion without access to surgical care, 1.7 billion are children and adolescents ${ }^{5}$. The vast majority of these children and adolescents live in LMICs, where the pediatric age group represents a disproportionately larger fraction of the population. In these settings, need for children's surgical care is high and poor care results in significant morbidity and mortality ${ }^{6}$. A child born in sub-Saharan Africa has an estimated $85 \%$ risk of requiring surgical care by age 15 years ${ }^{7}$. Improving surgical for children in LMICs can achieve several important goals, including early identification of correctable congenital anomalies and other surgical conditions, treatment of life-threatening injuries and burns, minimizing acute and longterm suffering, protecting families from substantial economic losses, and increasing economic productivity. For many childhood diseases including congenital anomalies, cancer, injuries and other emergencies, surgery remains the only curative modality in low resource regions of the world. Surgical care for children also has a role in achieving health-related Sustainable Development Goals and targets, in particular 1) Ending preventable deaths in newborns and 
children under five, 2) Reduction in death and disability for road traffic injuries and noncommunicable diseases, 3) Ensuring universal health coverage, and 4) Scaling up the health workforce ${ }^{8}$.

The Global Initiative for Children's Surgery (GICS) is a consortium of providers, institutions, and allies from both resource-rich and poor settings, who envision a future where every child will have access to surgical care ${ }^{9}$. GICS includes representation from 13 pediatric specialties: anesthesia, orthopedic surgery, neurosurgery, plastic surgery, urology, cardiac surgery, critical care, general pediatric surgery, otolaryngology, radiology, ophthalmology, oral and maxillofacial surgery and nursing. The goals of GICS are to: 1) promote the inclusion of children's surgical care within existing surgical and global child health initiatives; 2) provide strategic direction among the many children's surgery sub-specialties; and 3) identify highpriority needs and solutions from the perspective of local providers.

At the inaugural GICS meeting in London, England (May 2016) providers from LMICs expressed the need for guidelines that could be adapted for use in their local environment, especially for the purpose of advocating for increased resources. The Optimal Resources for Surgical Care (OReCS) project thus evolved to provide guidelines for essential surgical care for children in LMICs. A document was drafted and refined at the second and third GICS meetings (October 2016 and January 2018) with ongoing specialty expert input through multiple working groups. The OReCS document is divided into two parts: Part 1: Guidelines for Different Levels of Care, and Part II: Supplies, Equipment and Infrastructure. Both are included as Supplementary Material. The OReCS document outlines the resources required to care for children with surgical conditions at all levels of the health care system (Table 1). The resources needed for different levels of children's surgical care are outlined in the form of templates, which are designed to support basic, intermediate and complex/advanced surgical care for children in low resource settings. The goal of these templates is to provide a guide for minimum standards for those planning surgical services for children in low-resource settings. Thus, the OReCS 
document provides pragmatic, yet comprehensive recommendations regarding the resources, training and research priorities required for safe and high-quality surgical services for children in LMICs.

In conclusion, GICS convened a meeting of key stakeholders, led by LIMIC surgical and anesthesia providers, to develop guidelines for emergency and essential surgical services for children. The resulting product was the OReCS document which describes the resources needed to care for children with surgical problems at all levels of the health care system in LMICs. The document provides a strategy for integrating children's surgical care into national surgery, obstetric and anesthesia plans, or for developing surgical services within a child health policy/plan. The OReCS document can also serve as an important starting point for gap analysis, quality improvement, hospital assessment, training curricula, and for advocacy for children's surgical care. The OReCS program provides and important opportunity to mobilize the diverse community of international stakeholders to both implement and integrate children's surgery into existing and future global health initiatives.

\section{Electronic supplementary material}

Optimal Resources for Children's Surgical Care: I. Guidelines for Different Levels of Care Optimal Resources for Children's Surgical Care: II. Supplies Equipment and Infrastructure

\section{Author contributions}

Writing, editing and formatting of the manuscript were performed by DG, EA, DO, KO and SB. Editing of the manuscript, critical mentorship and guidance, as well as development of the GICS organization was accomplished by EA, TB, SB, MB, MC, ZG, PK, BK, RK, VM, KO, DO, DP, JS, LSG, SS, BY, KL and DF. Creation of the content, themes, projects and conclusions of the manuscript was undertaken by the membership of the Global Initiative for Children's Surgery in its entirety. 


\section{Funding}

The activities of the Global Initiative for Children's Surgery have been funded with support from the Archie Foundation, American Pediatric Surgery Association, American Society of Anesthesiologists, Association of Anaesthetists of Great Britain and Ireland, the British Association of Paediatric Surgeons, American College of Surgeons, Canadian Anesthesiologists' Society, Canadian Association of Paediatric Surgeons, Children's Hospital of Wisconsin, Family and Friends, Heart to Heart, International Society for Pediatric Neurosurgery, KidsOR, Mending Kids, Operation Smile, Orbis, Pacific Association of Pediatric Surgeons, Pediatric Orthopedic Society of North America, Royal College of Surgeons of England, Shriner's Hospital for Children of Northern California, Smile Train, Society for Pediatric Anaesthesia, Surgery for Children ONLUS, Wonder Work, World Federation of Associations of Pediatric Surgeons, Yantalo Foundation. This research was also supported by grant number R25TW011216 from the Fogarty International Center, National Institutes of Health (NIH). The program is supported by the U.S. Department of State's Office of the U.S. Global AIDS Coordinator and Health Diplomacy.

\section{Compliance with ethical standards}

\section{Conflict of interest}

All authors have no conflicts of interest to declare. 


\section{References}

1. Mock CN, Donkor P, Gawande A, Jamison DT, Kruk ME, Debas HT. Essential surgery: key messages from Disease Control Priorities, 3rd edition. Lancet 2015; 385(9983): 2209-19.

2. Meara JG, Leather AJ, Hagander L, et al. Global Surgery 2030: evidence and solutions for achieving health, welfare, and economic development. Lancet 2015.

3. Strengthening emergency and essential surgical care and anaesthesia as a component of universal health coverage. World Helath Organization 2015. Available from: http://apps.who.int/medicinedocs/documents/s21904en/s21904en.pdf (Accessed August 25, 2018) 4. Surgical Care Systems Strengthening: Developing national surgical, obstetric and anaesthesia plans. World Health Organization 2017. Available from: http://apps.who.int/iris/bitstream/handle/10665/255566/9789241512244-eng.pdf (Accessed August 25, 2018).

5. Mullapudi B, Grabski D, Ameh E, Ozgediz D, Thangarajah H, Kling K, Alkire B, Meara B, Bickler S. Estimates of number of children and adolescents without access to surgical care. Bull World Health Organ, In press.

6. Bickler SW, Rode H. Surgical services for children in developing countries. Bull World Health Organ 2002; 80(10): 829-35.

7. Bickler SW, Telfer ML, Sanno-Duanda B. Need for paediatric surgery care in an urban area of The Gambia. Tropical doctor 2003; 33(2): 91-4.

8. Henry JA, Abdullah F. Global Surgical Care in the U.N. Post-2015 Sustainable Development Agenda. World J Surg 2016; 40(1): 1-5.

9. Goodman LF, St-Louis E, Yousef Y, et al. The Global Initiative for Children's Surgery: Optimal Resources for Improving Care. Eur J Pediatr Surg 2017. 


\section{Key Messages}

- Surgical care has an incontrovertible, crosscutting role in achieving child health.

- Children develop different surgical diseases compared to adults, present unique anesthetic challenges, and have special perioperative needs.

- The Optimal Resources for Children's Surgery document provides a strategy for integrating surgical care for children into National Surgical, Obstetric and Anesthesia Plans.

- There is an important opportunity to prevent death and reduce disability in children by scaling up surgical care in low- and middle-income countries. 